\title{
Using LHC to Discover the Bilepton
}

\section{Paul H. Frampton*}

Dipartimento di Matematica e Fisica "Ennio De Giorgi", Università del Salento and INFN-Lecce, Via Arnesano, 73100 Lecce, Italy.

E-mail: pau1.h.framptondgmai1.com

We discuss a minimal and simple extension of the standard electroweak model and discuss its uniqueness properties. Its most readily testable prediction is the existence of $|Q|=|L|=2$ gauge bosons, bileptons, which decay into like-sign lepton pairs. We discuss how a discovery could be made at the LHC.

Corfu Summer Institute 2019 "School and Workshops on Elementary Particle Physics and Gravity" (CORFU2019)

31 August - 25 September 2019

Corfù, Greece

${ }^{*}$ Speaker. 


\section{Introduction}

Because the two most popular theoretical models aiming beyond the standard model - electroweak supersymmetry and large extra dimensions - have received no encouragement from LHC data, in this talk we shall discuss what seems to be the most likely first BSM particle. It is now being searched for by the ATLAS Collaboration.

The Bilepton Model was invented as an example of a class of models which turned out, up to variants, to have one distinct member.

Speculation

Probability LHC will find a new particle : $2 / 3$ likely. If so, guess that the bilepton is $90 \%$ likely so overall the bilepton can be $(2 / 3) \times 90 \%$ which means $60 \%$ probable.

We shall not have time to explain how this model was invented historically but there is no Royal Road to model building. One generally aims for

(i) motivation usually by addressing a question unanswered within the Standard Model.

(ii) testability by explicit predictions.

Both (i) and (ii) are satisfied by the Bilepton Model.

Corfu Summer Institute 2019 "Schools and Workshops on Elementary Particle Physics and Gravity" (CORFU2019). 31 August - 25 September 2019. Corfu, Greece.

(c) Copyright owned by the author(s)under the terms of the Creative Commons. AttributionNonCommercial-NoDerivatives 4.0 International License (CC BY-NC-ND 4.0)

\section{Bilepton Model}

The gauge group is:

$$
S U(3)_{C} \times S U(3)_{L} \times U(1)_{X}
$$

The simplest choice for the electric charge is

$$
Q=\frac{1}{2} \lambda_{L}^{3}+\left(\frac{\sqrt{3}}{2}\right) \lambda_{L}^{8}+X\left(\frac{\sqrt{3}}{\sqrt{2}}\right) \lambda^{9}
$$

where

$$
\operatorname{Tr}\left(\lambda_{L}^{a} \lambda_{L}^{b}\right)=2 \delta^{a b}
$$

and

$$
\lambda^{9}=\left(\frac{\sqrt{2}}{\sqrt{3}}\right) \operatorname{diag}(1,1,1)
$$


Thus a triplet has charges $(X+1, X, X-1)$.

Leptons are treated democratically in each of the three families. They are colour singlets in antitriplets of $S U(3)_{L}$ :

$$
\begin{aligned}
& \left(e^{+}, v_{e}, e^{-}\right)_{L} \\
& \left(\mu^{+}, v_{\mu}, \mu^{-}\right)_{L} \\
& \left(\tau^{+}, v_{\tau}, \tau^{-}\right)_{L}
\end{aligned}
$$

All have $X=0$.

Quarks in the first family are colour triplets and left-handed triplets plus three singlets

$$
\left(u^{\alpha}, d^{\alpha}, D^{\alpha}\right)_{L} \quad\left(\bar{u}_{\alpha}\right)_{L},\left(\bar{d}_{\alpha}\right)_{L},\left(\bar{D}_{\alpha}\right)_{L}
$$

Similarly for the second family

$$
\left(c^{\alpha}, s^{\alpha}, S^{\alpha}\right)_{L} \quad\left(\bar{c}_{\alpha}\right)_{L},\left(\bar{s}_{\alpha}\right)_{L},\left(\bar{S}_{\alpha}\right)_{L}
$$

The $\mathrm{X}$ values are for the triplets are $X=-1 / 3$ and for the singlets $X=-2 / 3,+1 / 3,+4 / 3$ respectively. The electric charge of the new quarks $D, S$ is $-4 / 3$.

The quarks of the third family are treated differently. The color triplet quarks are here in a lefthanded antitriplet and three singlets under $S U(3)_{L}$

$$
\left(b^{\alpha}, t^{\alpha}, T^{\alpha}\right)_{L} \quad\left(\bar{b}_{\alpha}\right)_{L},\left(\bar{t}_{\alpha}\right)_{L},\left(\bar{T}_{\alpha}\right)_{L}
$$

The antitriplet has $X=+2 / 3$ and the singlets carry $X=+1 / 3,-2 / 3,-5 / 3$ respectively. The new quark $T$ has $Q=5 / 3$.

Before discussing the symmetry breaking to $S U(2)_{L} \times U(1)_{Y}$ and the resulting mass spectrum, we must explain the nontrivial anomaly cancellation of this model which can explain the occurrence of three quark-lepton families.

\section{Cancellation of Triangle Anomalies}

There are six triangle anomalies which are potentially troublesome; in a self-explanatory notation these are diophantine equations[四] $\left(3_{C}\right)^{3},\left(3_{C}\right)^{2} X,\left(3_{L}\right)^{3},(3 L)^{2} X, X^{3}, X$.

The QCD anomaly $\left(3_{C}\right)^{3}$ is absent because QCD is, as usual, vectorlike. $\left(3_{C}\right)^{2} X$ vanishes because the quarks are in nine color triplets with net $X=0$ and nine antitriplets also with net $X=0$. The pure $\left(3_{L}\right)^{3}$ anomaly vanishes because there is an equal number of $3_{L}$ and $3_{L}^{*} \cdot\left(3_{L}\right)^{2} X$ cancels because the 
leptons have $\mathrm{X}=0$ and the quarks are in six triplets $3_{L}$ with $X=-\frac{1}{3}$ and three antitriplets $3_{L}^{*}$ with $X=+\frac{2}{3}$.

The $X^{3}$ cancellation can be checked by a little algebra: the three quark families contribute, respectively, $+6+6-12=0$.

It is especially interesting that this anomaly cancellation takes place between families. Each individual family possesses nonvanishing

$\left(3_{L}\right)^{3},(3 L)^{2} X, X^{3}$ anomalies.

Only with the number of families a multiple of three does the overall anomaly vanish. The imposition of the experimental requirement of asymptotic freedom of QCD then dictates that the number of families be exactly three.

The anomaly equations provide a system of six simulataneous diophantine equations. Algebraic number theory suggests that such a system almost never has any solution.

The fact that one unique solution exists, that underlying the Bilepton Model. This is the main reason for optimism that this arrangment of gauge group, embedding of the SM, and assignment of chiral fermions to irreps may be the one chosen by Nature.

As Einstein said when queried about the 1919 light-bending result and what would he have thought if the experiments had disagreed with his general relativity theory: Then I would feel sorry for the Lord. The theory is correct. In our experience, such infinite self-belief exists in some of the best particle theorists.

\section{Upper Limit on Bilepton Mass}

The symmetry breaking to the standard model is achieved by a Vacuum Expectation Value (VEV) of an $X=+1$ triplet $\left\langle\Phi^{a}\right\rangle=U \delta^{a 3}$. This gives mass $\Lambda_{D, S, T} U$ to the new quarks $D, S, T$ where $\Lambda_{i}$ are the Yukawa couplings. It also provides mass to five gauge bosons: the bileptons $\left(Y^{ \pm \pm}, Y^{ \pm}\right)$and $Z^{\prime}$.

Electroweak breaking is achieved by VEVs of two triplets $<\phi^{a}>=v \delta^{a 2}$ (with $X=0$ ) and $<$ $\phi^{\prime a}>=v^{\prime} \delta^{a 1}$ (with $X=-1$ ) and a doublet VEV in a sextet with $X=0$

$<H^{\alpha \beta}>=y \sqrt{10}\left(\delta^{\alpha 1} \delta^{\beta 3}+\delta^{\alpha 3} \delta^{\beta 1}\right)$

We note that because of global $L$ symmetry, the $W^{+}$and $Y^{+}$do not mix. For the same reason, the new quarks with exotic charges $(D, S, T)$ have lepton numbers $(+2,+2,-2)$ respectively.

Let the scale of breaking $331 \rightarrow 321$ be $\mu$. To avoid imaginary coupling constants with $g_{i}^{2}<0$ which violate unitarity it is necessary to impose an upper limit on $\mu$ such that

$$
\sin ^{2} \theta(\mu) \leq \frac{1}{4}
$$


while at the $Z$ pole the value is

$$
\sin ^{2} \theta\left(M_{Z}\right) \simeq 0.231
$$

which increases using the renormalisation group to $\frac{1}{4}$ at $\mu \simeq 4 \mathrm{TeV}$. Adopting this leads to

$$
M_{Y^{ \pm \pm}} \leq 2 \mathrm{TeV}
$$

by analogy with the electroweak theory where $M\left(W^{ \pm}\right)=80 \mathrm{GeV}$ which is less than the $S U(2) \times$ $U(1) \rightarrow U(1)$ breaking scale which is $\sim 248 \mathrm{GeV}$.

The estimated upper limit on bilepton mass of $2 \mathrm{TeV}$ is good news for the ongoing LHC bilepton search.

\section{Lower Limit on Bilepton Mass}

Perhaps surprisingly the lower limit comes not from colliders but from two table-top experiments.

Concidentally both experiments have been done at PSI (= Paul Scherrer Institute). A second coincidence is they both give closely the same result for the bilepton lower mass bound.

Firstly there is $\mu^{+} e^{-} \rightarrow \mu^{-} e^{+}$which can be mediated by doubly-charged bilepton exchange. Called muonium-antimuonium conversion it provides $m_{Y^{ \pm \pm}}>800 \mathrm{GeV}$.

Secondly there is $\mu^{-} \rightarrow e^{-} v_{e} \bar{v}_{\mu}$ mediated by singly-charged bilepton exchange which by Fierz rearrangement is a $(V+A)$ contribution to $\mu^{-} \rightarrow e^{-} \bar{\mu}_{e} v_{\mu}$ whose Michel parameter $\xi$ in $(V-\xi A)$ is $1 \geq \xi>0.997$. This requires that $m_{Y^{ \pm}}>800 \mathrm{GeV}$.

\section{Bilepton Phenomenology at the LHC}

A study of bilepton pair production and two or more jets at the LHC with $\sqrt{s}=13 \mathrm{TeV}$ was made, using the Feynman rules for the Bilepton Model.

About 3000 tree-level Feynman graphs were implemented by SARAH 4.9.3.

Amplitudes were computed numerically by MadGraph.

Simulation of parton showers and hadronisation was made by HERWIG.

For more details, see[[, []] 


\section{Resonance Bumps}

One method [ [ 9 ] of estimating the numbers of events at ATLAS is to use old ATLAS data which were analysed to search for a SSM(=Sequential Standard Model) $Z^{\prime}$ which was not discovered. There is some similarity between production of $\mathrm{Y}$ and $Z^{\prime}$. The biggest difference is that $\mathrm{Y}$ must be pair produced so we approximate by using $\sigma_{S S M}^{Z^{\prime}}\left(M\left(Z^{\prime}=2 M(Y)\right)\right.$. We need to estimate the brancing ratio (BR) for $\left(Y \rightarrow e^{+} e^{+}, \mu^{+} \mu^{+}, \tau^{+} \tau^{+}\right)$. Because there exist non-leptonic decays $B \rightarrow Q \bar{q}, q \bar{Q}$ where $\mathrm{Q}$ is an exotic quark, the BRs depend on the mass $\mathrm{M}(\mathrm{Q})$ of the exotic quarks.

We have calculated the branching ratios into each flavour of like-sign lepton pairs, assuming 800 $\mathrm{GeV}$ as the common mass of the three exotic quarks, see Table 1. If some of the exotic quarks are more massive than $800 \mathrm{GeV}$ these BRs would be slightly larger so the estimates in Table 2 will be conservative lower limits on the event rates. 
Table 1: Branching ratios into like-sign lepton pairs for each of the three flavours of charged lepton $e, \mu, \tau$.

\begin{tabular}{||c|c||}
\hline $\begin{array}{c}\mathrm{M}(\mathrm{Y}) \\
\mathrm{GeV}\end{array}$ & $\begin{array}{c}\text { BR=branching ratio } \\
\text { into like-sign lepton pair } \\
\text { (per flavour) }\end{array}$ \\
\hline \hline 800 & 0.33 \\
\hline 1100 & 0.31 \\
\hline 1400 & 0.28 \\
\hline 1700 & 0.25 \\
\hline 2000 & 0.21 \\
\hline \hline
\end{tabular}

We re-calculated the numbers of signal events and SM background events, using the values of BR from Table 1. The results which are our most reliable predictions for the LHC are displayed in Table 2.

Table 2: Numbers of signal and background events at resonance in like-sign lepton pairs for integrated luminosity 150/fm. This Table gives our most reliable predictions.

\begin{tabular}{||c|c|c|c|c||}
\hline $\begin{array}{c}\mathrm{M}(\mathrm{Y}) \\
\mathrm{GeV}\end{array}$ & $\begin{array}{c}\sigma_{S S M}^{Z^{\prime}}\left(M_{Z^{\prime}}=2 M(Y)\right) \\
(\mathrm{fb})\end{array}$ & $(B R)^{2}$ & $\begin{array}{c}\text { Signal } \\
\text { events }\end{array}$ & $\begin{array}{c}\text { Background } \\
\text { events. }\end{array}$ \\
\hline \hline 800 & 100 & 0.109 & 1635 & $<0.01$ \\
\hline 1100 & 20 & 0.096 & 288 & $<0.01$ \\
\hline 1400 & 6 & 0.078 & 70 & $<0.01$ \\
\hline 1700 & 1 & 0.062 & 9 & $<0.01$ \\
\hline 2000 & 0.6 & 0.044 & 4 & $<0.01$ \\
\hline \hline
\end{tabular}

\section{Summary}

Parity violation in weak interactions, chiral fermions and triangle anomalies underly the Standard Model and its extension to the Bilepton Model.

A possible search for ATLAS and CMS is for $Y^{ \pm \pm}$which underly an explanation of three families in the Bilepton Model predicts doubly-charged siblings $Y^{ \pm \pm}$to accompany $W^{ \pm}$.

There is the expected mass range

$800 \mathrm{GeV} \leq M_{Y^{ \pm \pm}} \leq 2000 \mathrm{GeV}$ 
which renders this new particle accessible to the LHC.

What is encouraging about the Bilepton Model at the LHC is that our calculations of the new signal and the Standard Model background suggest that obtaining a $5 \sigma$ statistically significant signal for the resonance seems, at least to a theorist, to be straightforward.

As may be recalled from the 2012 announcement by CERN about discovery of the Higgs Boson, $5 \sigma$ is their official requirement for announcing the discovery of a new particle, as appears readily possible for the bilepton. Discovery of the bilepton would be more transformative than the Higgs Boson since the latter is already contained in the Standard Model. One well-known precedent of a particle discovery was the $\Omega^{-}$particle discovered at Brookhaven National Laboratory in 1964 which confirmed Gell-Mann's Eightfold Way theory proposed in 1961. That experimental confirmation thus took only three years. If it were discovered in 2020, the bilepton would have taken 28 years from 1992 but recall, however, that discovery of the Higgs boson took much longer.

\section{References}

\section{References}

[1] P.H. Frampton, Chiral Dilepton Model and the Flavor Question. Phys. Rev. Lett. 69, 2889 (1992).

[2] G. Corcella, C. Corianò, A. Costantini and P.H. Frampton, Bilepton Signatures at the LHC. Phys. Lett. B773, 544 (2017). arXiv:1707.01381[hep-ph].

[3] G. Corcella, C. Corianò, A. Costantini and P.H. Frampton, Exploring Scalar and Vector Bileptons at the LHC in the 331 Model. Phys. Lett. B785, 73 (2018.) arXiv:1806.04536 [hep-ph] .

[4] C. Corianò and P.H. Frampton, Possible Bilepton Resonances in Like-Sign Pairs. Mod.Phys.Lett. A33, 1950076 (2019). arXiv:1812.02723 [hep-ph] . 